\title{
Epigenetic regulation of the embryonic oncogene ERas in gastric cancer cells
}

\author{
MASAKAZU YASHIRO ${ }^{1,2}$, KOICHI YASUDA ${ }^{1}$, TAKAFUMI NISHII $^{1}$, RYOJI KAIZAKI $^{1}$, \\ TETSUJI SAWADA ${ }^{1}$, MASAICHI OHIRA ${ }^{1}$ and KOSEI HIRAKAWA ${ }^{1}$ \\ ${ }^{1}$ Department of Surgical Oncology, ${ }^{2}$ Oncology Institute of Geriatrics and Medical Science, Osaka City University \\ Graduate School of Medicine, 1-4-3 Asahi-machi, Abeno-ku, Osaka 545-8585, Japan
}

Received June 5, 2009; Accepted July 29, 2009

DOI: 10.3892/ijo_00000414

\begin{abstract}
ERas is a recently identified oncogene that supports the tumorigenic growth of embryonic stem cells, it is constitutively active in the absence of mutation. ERas oncogene is expressed only in viviparity phase cells, but not in somatic cells because of epigenetic transcriptional silencing in the somatic phase. The aim of this study was to clarify the ERas expression and its epigenetic regulation in gastric cancer of somatic phase. Fifteen gastric cancer cell lines were used. ERas mRNA expression and its epigenetic regulation were examined by reverse transcription-polymerase chain reaction and bisulfite sequencing analysis. To identify a subset of cancer stem cells, termed 'side population' (SP) cells, flow cytometry analysis was performed. ERas is expressed in 8 of the 15 gastric cancer cell lines, but is silenced in the remaining 7 cancer cell lines and normal cell lines. Six of 7 cancer cell lines without ERas expression had promoter methylation, which correlated with silencing of ERas expression. ERas expression is re-activated following treatment with the DNA methyltransferase inhibitor 5-aza$\mathrm{CdR}$. The percentage of SP fraction of ERas-positive gastric cancer cells was significantly $(\mathrm{p}=0.024)$ higher $(3.4 \pm 1.8 \%)$, in comparison to that of ERas-negative cells $(1.6 \pm 0.4 \%)$. These findings suggested that the activating ERas oncogene might be associated with tumorigenic growth of somatic cells, and might be a putative molecule responsible for cancer stem celllike characteristics in gastric cancer. Loss of methylation in the promoter of ERas might be one of mechanisms responsible for the re-expression of an embryonic oncogene in gastric cancer.
\end{abstract}

\section{Introduction}

Embryonic stem (ES) cells share many growth properties with tumor cells. There might be some common molecular

Correspondence to: Dr Masakazu Yashiro, Department of Surgical Oncology, Osaka City University Graduate School of Medicine, 1-4-3 Asahi-machi, Abeno-ku, Osaka 545-8585, Japan

E-mail:m9312510@med.osaka-cu.ac.jp

Key words: ERas oncogene, promoter methylation, gastric carcinoma, cancer stem cells mechanisms between ES cells and tumor cells. A teratoma, which is composed of all three germ layers, is formed when ES cells are subcutaneously injected into immunodeficient or isogenic mice. ERas was recently identified as an oncogene that supports this tumor-like propagation of ES cells (1). ERas product is a constitutively active Ras protein in the absence of mutation (2), while conventional ras oncogenes, $\mathrm{K}-r a s, \mathrm{~N}-r a s$ and $\mathrm{H}-r a s$, acquire activation of carcinogenesis by point mutation. The phosphatidylinositol-3-OH kinase cascade is important in the growth-promoting activity of ERas, but not with Raf. ERas oncogene is expressed only in viviparity phase cells, but not in somatic cells because of epigenetic regulation of ERas oncogene in the somatic phase. Epigenetic regulation of tumor-associated gene is a common motif in many types of carcinomas (3). ERas expression in cancer might also be regulated by epigenetic modification.

Tumor-associated transcriptional silencing is most frequently described in association with epigenetic mechanisms $(4,5)$. DNA methylation is a common epigenetic mechanism implicated in the transcriptional silencing of genes, and plays an important role in human carcinogenesis. The process of hypermethylation is carried out by DNA methyltransferase, which catalyzes the covalent addition of a methyl group from a donor $S$-adenosylmethionine to the 5 position of cytosine, predominantly within the $\mathrm{CpG}$ dinucleotide (6). DNA methyltransferases, such as Dnmt1, Dnmt3a, and Dnmt3b, are depleted by being bound to their inhibitors and are thereby unavailable for methylation, resulting in significant demethylation after repeated replication (7). The DNA methyltransferase inhibitor, 5-aza-2'-deoxycytidine (5-aza-CdR), has been shown to reverse the hypermethylation status of promoters, allowing re-expression of silenced genes.

Recently, the concept of cancer stem cell (CSC) was reported to be responsible for self-renewing, differentiation, tumorigenesis and to contribute to some individual resistance to cancer therapy (8-11). CSC has been isolated from several human tumors that have markers for putative normal stem cells, including gastric cancer (6). Previous studies have shown that adult stem cells can be identified by a side population (SP) phenotype. The SP, first described by Goodell et al (12), is a small subpopulation of cells with enriched stem cell activity that shows a distinct low Hoechst 33342 dye staining pattern. Concurrent studies have shown 
SP cells in human cancers of different origins (8-11). These studies have suggested that the SP may be a source of CSCs. Then we hypothesized that ERas oncogene might be one of the markers for CSC-like characteristics.

We recently described the up-regulation of ERas by histone acetylation in various carcinoma cells with the silencing of the ERas oncogenes (13). Here, we report the expression of ERas gene and epigenetic regulation by DNA methylation of the promoter lesion in human gastric cancer cells, and the significant correlation between the expression of ERas gene and CSC-like SP cells.

\section{Materials and methods}

Cell culture and cell lines. The culture medium was composed of Dulbecco's modified Eagle's medium (DMEM; Nikken Bio., Kyoto, Japan) with addition of $2 \%$ fetal calf serum (FCS; Life Technologies, Grand Island, NY), $100 \mathrm{IU} / \mathrm{ml}$ of penicillin (ICN Biomedicals, Aurora, $\mathrm{OH}$ ), $100 \mu \mathrm{g} / \mathrm{ml}$ of streptomycin (ICN Biomedicals), and $0.5 \mathrm{mM}$ of sodium pyruvate (Cambrex, Wakersville, MD). Fifteen gastric cancer cell lines, NUGC-2, NUGC-3, NUGC-4, OCUM-1, OCUM-2M (14), OCUM-2MD3 (15), OCUM-6 (16), OCUM-8 (17), OCUM-9, OCUM-12, MKN-7, MKN-45, MKN-74, FU-97, and KATO-III, and 3 normal cell lines including 2 fibroblast cell lines, NF-24 and NF-26, and mesothelial cells, were used in this study. OCUM-1, OCUM-2M, OCUM-2MD3, OCUM-6, OCUM-8, OCUM-9, OCUM-12 and normal cell lines were established in our laboratory. Other human carcinoma cell lines were obtained from the JCRB cell bank (Osaka, Japan) or the American Type Culture Collection (Rockville, MD). The cells were seeded in a 100mm dish (Falcon, Lincoln Park, NJ) and cultured in $10 \mathrm{ml}$ of medium at $37^{\circ} \mathrm{C}$ in a humidified atmosphere containing $5 \%$ $\mathrm{CO}_{2}$ in air.

Reverse-transcription polymerase chain reaction (RT-PCR). Total cellular RNA was extracted from 15 gastric cancer cell lines and normal cell lines with TRIzol (Life Technologies) according to the manufacturer's protocol. cDNAs were synthesized with an M-MLV-RT kit (Life Technologies) using oligo hexamers. Relevant cDNAs were amplified by PCR for 35 cycles with Taq DNA polymerase (Nippon Gene, Tokyo, Japan) on a thermal cycler. The following ERas primers and Dnmt primers were used: ERas, 5'-ACCACGACCCCAT CCA-3' and 5'-GGACCAGCAGGGAAAAGGC-3'; Dnmt1, 5'-GGCTTGGGCAGCCTATGAA and 5'-GGAATGACCG AGACGCAGTC; Dnmt3a, 5'-CAGCGTCACACAGAAGC ATATCC and 5'-GGTCCTCACTTTGCTGAACTTGG; Dnmt3b, 5'-CCTGCTGAATTACTCACGCCCC and 5'-GTC TGTGTAGTGCACAGGAAAA; and $18 S$ rRNA, 5'-TCAAG AACGAAAGTCGGAGG and 5'-GGACATCTAAGGGCAT CACA. PCR conditions were as follows: predenaturation, $94^{\circ} \mathrm{C}$ for $10 \mathrm{~min}$; denaturation, $94^{\circ} \mathrm{C}$ for $50 \mathrm{sec}$; annealing, $59^{\circ} \mathrm{C}$ for $50 \mathrm{sec}$; extension, $72^{\circ} \mathrm{C}$ for $1 \mathrm{~min}$; and final incubation, $72^{\circ} \mathrm{C}$ for $7 \mathrm{~min}$. PCR products of ERas were $383 \mathrm{bp}$ in length. These products were then applied to a $2 \%$ agarose gel and electrophoresed. As an internal control, RT-PCR for glyceraldehyde-3-phosphate dehydrogenase $(G A P D H)$ was performed: sense primer, 5'-ACCTGACCTG
CCGTCTAGAA-3', and antisense primer, 5'-TCCACCACC CTGTTGCTGTA-3'. PCR conditions were as follows: predenaturation, $94^{\circ} \mathrm{C}$ for $5 \mathrm{~min}$; denaturation, $94^{\circ} \mathrm{C}$ for $30 \mathrm{sec}$; annealing, $60^{\circ} \mathrm{C}$ for $30 \mathrm{sec}$; extension, $72^{\circ} \mathrm{C}$ for $1 \mathrm{~min}$; and final incubation, $72^{\circ} \mathrm{C}$ for $1 \mathrm{~min}$.

DNA methyltransferase inhibitor treatment. Cell lines were treated with varying doses of 5-aza-CdR (Sigma, St. Louis, MO), a specific inhibitor of DNA methyltransferase, ranging from 2 to $10 \mu \mathrm{M}$ for various times (18).

$K$-ras mutation analysis. To reveal relations between ERas expression and K-ras mutations, K-ras mutation analysis was performed by dot blot analysis as previously described (19). PCR amplification of exon 1 of K-ras containing codons 12 and 13 was performed. The 104-bp amplicons were dotblotted onto nylon filters (Hybond-N; Amersham, Bukinghamshire, UK) and hybridized with radiolabeled oligomer primers representing all possible mutations at codon 12 and the GAC mutation of codon 13 .

Bisulfite sequencing analysis. Genomic DNA of the cell lines $\left(1 \times 10^{7}\right)$ was extracted with phenol-chloroform. We treated the samples with sodium bisulfite, which converts nucleotides $\mathrm{C} \rightarrow \mathrm{T}$ in the absence of methylation. In contrast, once methylation occurs at the $\mathrm{C}$ residues, they will resist the treatment. Sodium bisulfite modification of $4 \mu \mathrm{g}$ genomic DNA was carried out using the EZ DNA Methylation kit (Zymo Research, Orange, CA). Bisulfite sequencing was performed using bisulfite-modified DNA. For ERas gene, a CpG-rich region (between positions -251 and +94 relative to transcriptional start site) was amplified by PCR for 35 cycles with Taq DNA polymerase on a thermal cycler. The following ERas primers for the bisulfite sequencing analysis were used: sense primer, 5'-AGGAGGGAAGGAGAGATGG-3' and antisense primer, 5'-ACCCACCACCACAACCTT-3'. PCR conditions were as follows: predenaturation, $94^{\circ} \mathrm{C}$ for $10 \mathrm{~min}$; denaturation, $94^{\circ} \mathrm{C}$ for $50 \mathrm{sec}$; annealing, $59^{\circ} \mathrm{C}$ for $50 \mathrm{sec}$; extension, $72^{\circ} \mathrm{C}$ for $1 \mathrm{~min}$; and final incubation, $72^{\circ} \mathrm{C}$ for $7 \mathrm{~min}$. The DNA templates were sequenced using Big Dye terminators (version 3.1). PCR products were analyzed using a genetic analyzer (ABI PRISM 310; Applied Biosystems, Foster City, CA) according to the manufacturer's instructions. Cytosine residues at non-CpG sites are converted to uracil by bisulfite treatment, whereas those at $\mathrm{CpG}$ sites remain as cytosine (if methylated) or are converted to uracil (if unmethylated). The fractional methylation was determined at each $\mathrm{CpG}$ site. Universal methylated DNA (Chemicon, Temecula, CA) was used as positive control.

Flow cytometry for SP analysis. The cell suspensions were labeled with Hoechst 33342 dye (Sigma, Osaka, Japan) using the methods described by Goodell et al (12) with modifications. Briefly, cells were removed, pelleted by centrifugation, washed with phosphate-buffered saline (PBS; Nikken Bio.), and resuspended at $2 \times 10^{6 /} \mathrm{ml}$ in prewarmed DMEM with $2 \%$ FCS (Life Technologies). The DNA binding dye, Hoechst 33342 (Sigma), was then added at a final concentration of $5 \mu \mathrm{g} / \mathrm{ml}$ and incubated for $60 \mathrm{~min}$ in the dark with interval mixing. The cells were washed, $1 \mu \mathrm{g} / \mathrm{ml}$ propidium iodide (Sigma) 

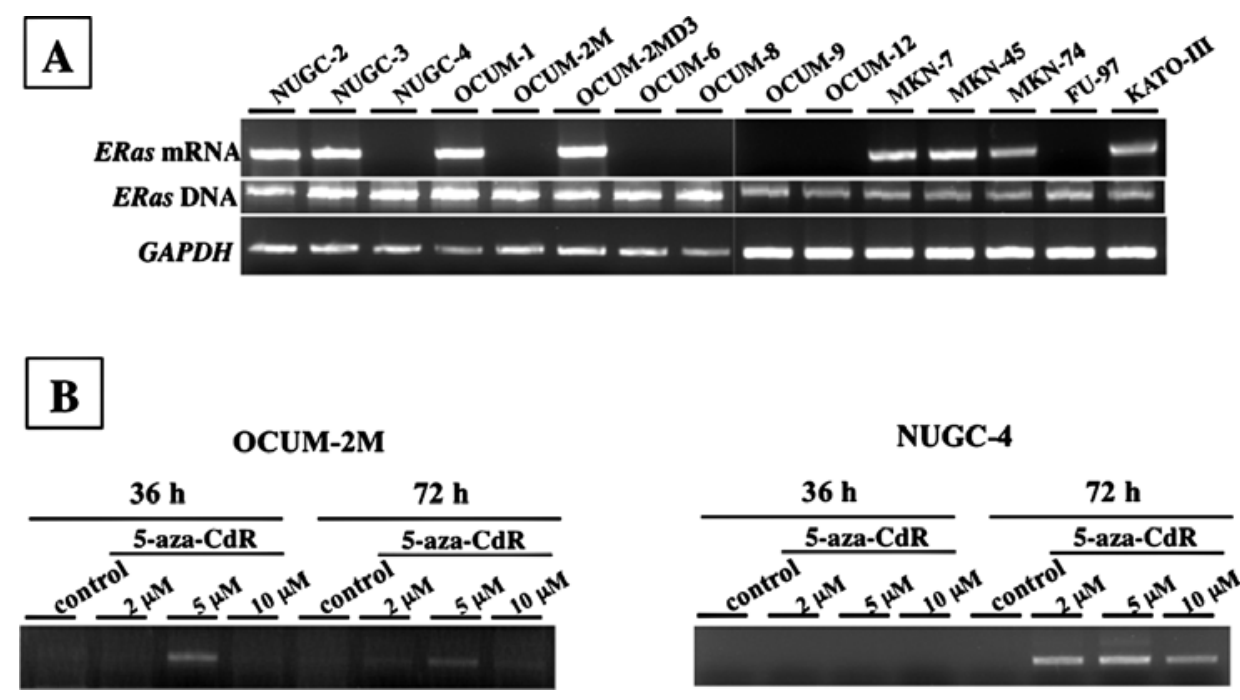

$\mathbf{C}$

OCUM-2M (5-aza-CdR)
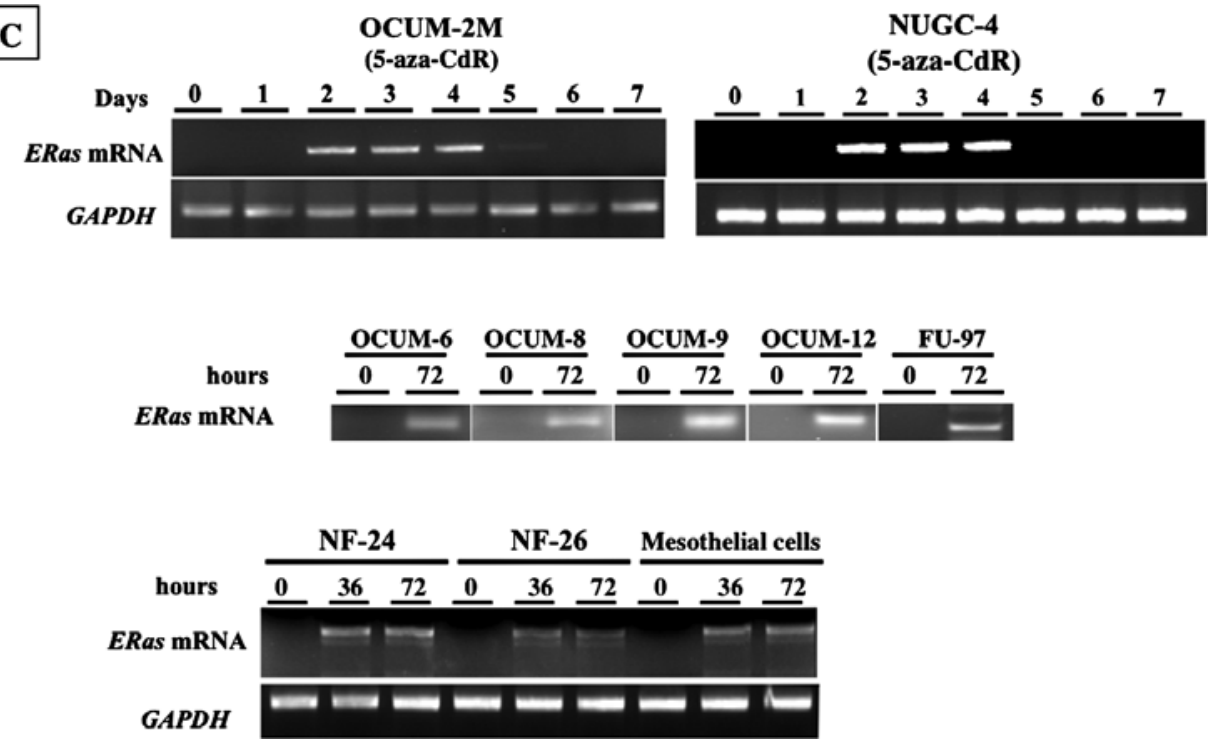

Figure 1. Expression of ERas mRNA in cell lines. (A), ERas mRNA was found in 8 (NUGC-2, NUGC-3, OCUM-1, OCUM-2MD3, MKN-7, MKN-45, MKN-74, and KATO-III) of 15 gastric cancer cell lines, while ERas DNA was found in all gastric cancer cell lines. (B), Determination of the optimal dose and duration of exposure of cell lines to 5-aza-CdR for ERas gene expression. ERas mRNA was expressed by 5-aza-CdR at 2-10 $\mu \mathrm{M}$ from 36 or $72 \mathrm{~h}$ in OCUM-2M and NUGC-4 cells. (C), Epigenetic regulation of ERas. ERas mRNA was expressed by $5 \mu \mathrm{M}$ of 5 -aza-CdR in OCUM-2M, NUGC-4, and 3 of 4 normal cell lines (NF-24, NF-26 and mesothelial cells).

was added, and the cells were kept at $4^{\circ} \mathrm{C}$ in the dark before FACS (FACSDiva Option, Becton-Dickinson, Mountain View, CA) dual-wavelength analysis. Because Hoechst 33342 extrudes from cells using verapamil-sensitive ABC transporters, a subset of the cells were incubated with $500 \mu \mathrm{mol} / 1$ verapamil for $30 \mathrm{~min}$ at $37^{\circ} \mathrm{C}$ before adding Hoechst 33342 to determine whether this would block the fluorescent efflux of SP cells in cancer cell lines. The Hoechst dye was excited at $350 \mathrm{~nm}$, and its fluorescence was measured at two wavelengths using a 450 DF10 (450/20 nm band-pass filter) and a 675LP (675 nm long-pass edge filter) optical filter. The gating on forward and side scatter was not stringent, and only debris was excluded.

Statistical analysis. The quantitative ratios of different groups were compared using Student's t-test. The Fisher's exact test was used to determine the significance of the difference between the covariates. Probability values of $p<0.05$ were regarded as statistically significant.

\section{Results}

ERas expression in gastric cancer. The expression of ERas mRNA was found 8 of 15 gastric cancer cell lines, while ERas DNA was found in all gastric cancer cell lines (Fig. 1A). The remaining 7 of 15 gastric cancer cell lines did not express ERas mRNA.

Effect of DNA methyltransferase inhibitor on ERas expression. We examined the effect of DNA methyltransferase inhibitor on the expression of ERas mRNA in 7 gastric cancer cell lines. We determined the optimal dose and timing for gene 
Table I. Relation between ERas expression, promoter methylation, K-ras mutation, and percentage of SP fraction in gastric carncer cell lines.

ERas mRNA expression

\begin{tabular}{|c|c|c|c|c|c|}
\hline Cell line & No treatment & 5-aza-CdR $(5 \mu \mathrm{M})$ & CpG promoter methylation & K-ras mutation & SP fraction $(\%)$ \\
\hline NUGC-2 & + & + & Unmethyl & wt & ND \\
\hline NUGC-3 & + & + & NI & wt & 2.41 \\
\hline NUGC-4 & - & + & Methyl & NI & 1.36 \\
\hline OCUM-1 & + & + & Unmethyl & wt & 2.47 \\
\hline OCUM-2M & - & + & Methyl & wt & 1.40 \\
\hline OCUM-2MD3 & + & + & Unmethyl & wt & 6.00 \\
\hline OCUM-6 & - & + & Methyl & wt & 1.90 \\
\hline OCUM-8 & - & + & Methyl & wt & 1.13 \\
\hline OCUM-9 & - & + & Methyl & wt & 1.93 \\
\hline OCUM-12 & - & + & Methyl & wt & 2.14 \\
\hline MKN-7 & + & + & NI & wt & 1.66 \\
\hline MKN-45 & + & + & Unmethyl & wt & 6.21 \\
\hline MKN-74 & + & + & Unmethyl & 12Asp & 2.71 \\
\hline FU-97 & - & + & NI & wt & 1.13 \\
\hline KATOIII & + & + & $\mathrm{NI}$ & wt & 2.51 \\
\hline
\end{tabular}

5-aza-CdR, 5-aza-2'-deoxycytidine. 12Asp, codon 12 aspartic acid; wt, wild-type. +, ERas mRNA expression positive; -, no ERas mRNA expression. Methyl, CpG methylation; unmethyl, unmethylation. ND, not determined. NI, not informative. SP, side population.

expression studies by characterizing the time course and dose response of treatment by 5 -aza-CdR in two cancer cell lines of ERas-negative expression, OCUM-2M and NUGC-4. The expression of ERas was found by 5 -aza-CdR at $2-10 \mu \mathrm{M}$ in both cell lines (Fig. 1B). The expression of ERas was found by 5 -aza-CdR treatment from $36 \mathrm{~h}$ or $72 \mathrm{~h}$ in both gastric cancer and normal cell lines. We then chose a standard dosing regimen of 5 -aza-CdR at $5 \mu \mathrm{M}$ for $72 \mathrm{~h}$. Effect of 5-aza-CdR on the ERas expression was examined in the 7 cancer cell lines without ERas mRNA expression (Fig. 1C). 5-aza-CdR treatment resulted in ERas expression in all 7 gastric cancer cell lines with respect to the silencing of ERas (Table I).

Methylation status of the ERas in gastric cancer. ERas gene contains a $\mathrm{CpG}$-rich region between positions -251 and +94 relative to transcriptional start site. The results of the methylation study were confirmed by bisulfite DNA sequencing. DNA direct sequencing analysis at the $5^{\prime}$ end promoter region of ERas, which contains 12 CpGs. Fig. 2A shows the results of a typical DNA sequencing with or without bisulfite treatment in normal NF-24 cells. Fig. 2B shows the results of a typical bisulfite DNA sequencing in gastric cancer cell lines, OCUM-2M, NUGC-4, and universal DNA. There was a ' $\mathrm{C}$ ' peak at the $\mathrm{CpG}$ sites at nucleotide position -84 , indicating partial methylation in OCUM-2M, NUGC-4 cells, where no ERas mRNA band was observed. In contrast, there was a ' $\mathrm{T}$ ' peak at the $\mathrm{CpG}$ sites at nucleotide -84 , indicating unmethylation in OCUM-2MD3, OCUM-1 cells, where ERas mRNA was expressed. The $\mathrm{CpG}$ sites were completely unmethylated (Fig. 2C). CpG methylation at
ERas promoter region was significantly more frequent in ERas-negative gastric cancer cell lines than in ERas-positive cell lines ( $p=0.002$; Fisher's exact test). The $\mathrm{CpG}$ methylation pattern correlated well with the down-regulated ERas mRNA expression in the cell lines (Table I).

Correlation between the expression of ERas and DNA methyltransferases. The expression of DNA methyltransferases, Dnmt1, Dnmt3a, and Dnmt3b, was different among the 15 gastric cancer cell lines (Fig. 2D). Decreased expression of Dnmt1, Dnmt3a, or Dnmt3b was recognized in NUGC-2, OCUM-2M, OCUM-2MD3, OCUM-6, OCUM-8, and OCUM-9 cells. However, no correlation was found between the ERas expression and the DNA methyltransferase expression.

Correlation between the ERas expression and the percentage of SP fraction in gastric cancer cell lines. We examined the existence of SPs in human gastric cancer cell lines by staining them with Hoechst 33342 dye to generate a Hoechst blue-red profile. SP phenotype was determined in human gastric cancer cell lines except for NUGC-2. Representative results analyzed by flow cytometry are shown in Fig. 3A. Most of the gastric cancer cell lines contain a component of cancer SP cells, ranging from $1.1 \%$ (FU-97) to $6.2 \%$ (MKN45) of gated cells, which decreased by treatment with verapamil (Fig. 3A). The percentage of SP fraction of ERaspositive gastric cancer cell lines $(3.42 \pm 1.8 \%)$ was significantly $(\mathrm{p}=0.024)$ higher compared to that of ERasnegative lines (1.57 $\pm 0.4 \%$; Fig. 3B). 
A

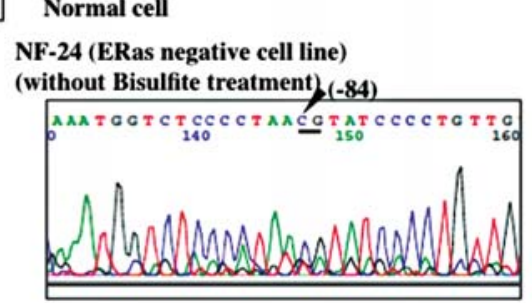

NF-24

(Bisulfite treatment)

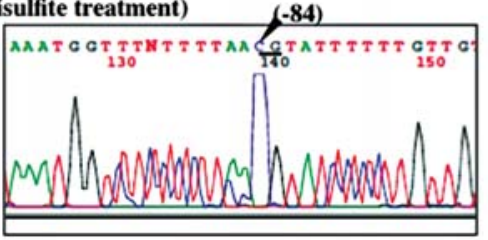

B Methylated CpG

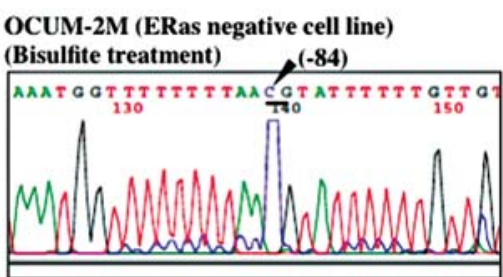

NUGC-4 (ERas negative cell line)

(Bisulfite treatment)

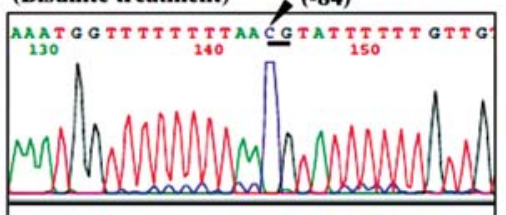

Universal DNA

(Bisulfite treatment) $(\mathbf{- 8 4})$

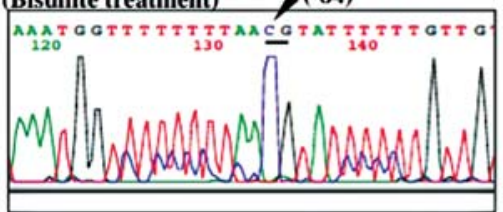

C Unmethylated CpG OCUM-2MD3 (ERas positive cell line) (Bisulfite treatment)

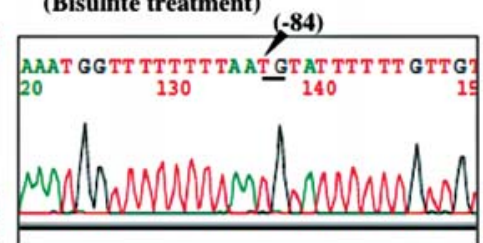

OCUM-1 (ERas positive cell line)

(Bisulfite treatment)

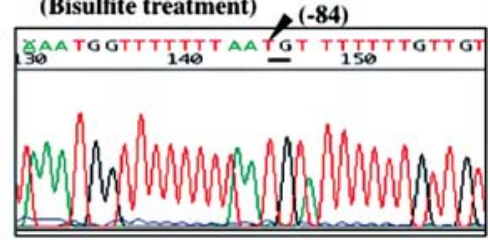

D

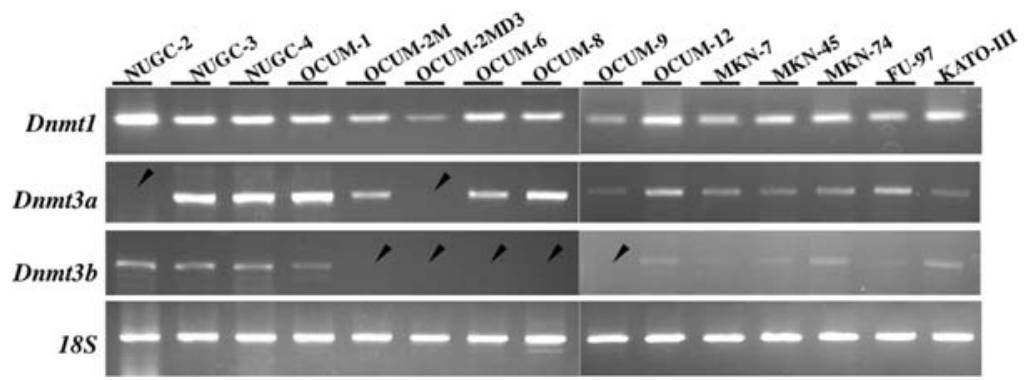

Figure 2. CpG methylation pattern of the ERas promoter in gastric cancer cells. (A), DNA sequencing with or without bisulfite treatment in the normal NF-24 cells. Partial methylation was found at -84 (arrow). (B), Bisulfite DNA sequencing of partially methylated cell lines, OCUM-2M, NUGC-4, and universal DNA. (C), Bisulfite DNA sequencing of the unmethylated cell lines OCUM-2MD3, OCUM-1. (D), The expression of DNA methyltransferase mRNA. The expression of DNA methyltransferases Dnmt3a or Dnmt3b, was decreased in 6 gastric cancer cell lines (arrows). Dnmt3a expression was decreased in NUGC-2 and OCUM-2MD3 cells. Dnmt3b expression was decreased in OCUM-2M, OCUM-2MD3, OCUM-6, OCUM-8, and OCUM-9 cells.

$\mathbf{A}$

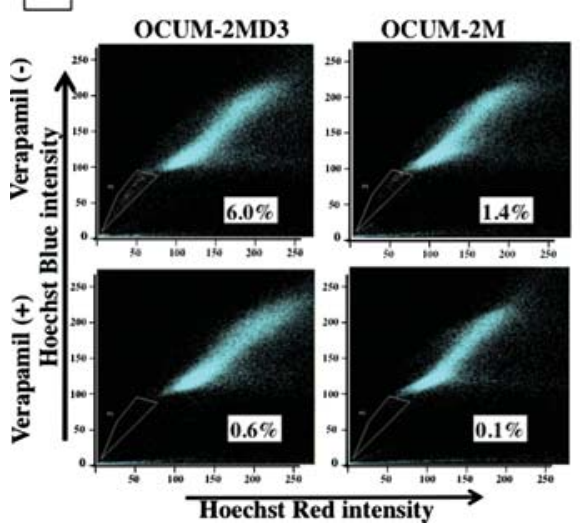

B

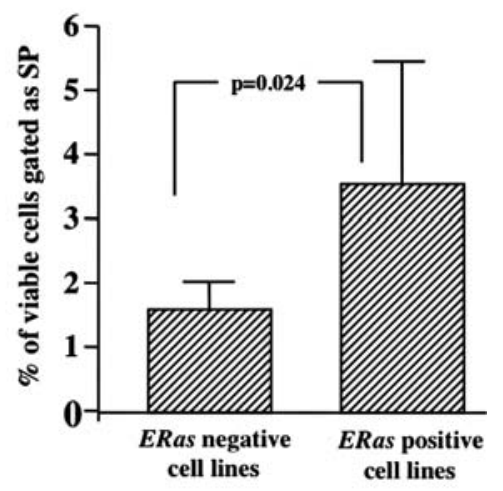

Figure 3. Analysis of side population (SP) cells in gastrointestinal cancers. (A), Representative SP cell isolation from various human gastrointestinal system cancer cell lines. OCUM-2MD3 and OCUM-2M cells were stained with Hoechst 33342 dye in the presence (upper panel) or absence (bottom panel) of verapamil and analyzed by flow cytometry. The percentage of SP fraction of OCUM-2MD3, an ERas-positive gastric cancer cell line, was 6.0\%, while that of OCUM-2M, an ERas-negative gastric cancer cell line, was $1.4 \%$. The SP, which disappears in the presence of verapamil, was gated and shown as a percentage of the whole viable cell population. Square, portion of SP. (B), The percentage of SP fraction of ERas-positive gastric cancer cell lines $(3.42 \%)$ was significantly ( $\mathrm{p}=0.024)$ higher compared to the control $(1.57 \%)$. The quantitative ratios of different groups were compared using Student's t-test. 
Correlation between the K-ras mutation and ERas expression. $\mathrm{K}$-ras mutations were found in only one of 14 cancer cell lines. No relation was found between ERas expression and $\mathrm{K}$-ras mutation in these cancer cell lines (Table I).

\section{Discussion}

ERas was reported to be expressed specifically in ES cells but not in normal somatic tissues (1). In this study, the constitutively active expression of ERas oncogene was found in 8 of 15 gastric cancer cell lines. These findings suggest that ERas oncogene might be associated with the tumorigenic growth of some somatic cells in gastric cancers. In contrast, $\mathrm{K}$-ras mutations were found in only one of 14 cancer cell lines. ERas signaling might be more closely associated with the progression of gastric carcinomas than K-ras signaling.

Silenced ERas oncogene was re-expressed under DNA methyltransferase inhibitor treatment in 3 normal and 7 cancer cell lines. The direct sequencing analysis showed the occurrence of methylation at the promoter $\mathrm{CpG}$ by showing unchanged $\mathrm{C}$ residues in 7 cell lines without ERas mRNA expression. The methylation correlated well with the down-regulated ERas mRNA expression in cell lines. ERas mutation was not found in any of the 15 gastric cancer cell lines (data not shown). These results suggested that the ERas expression in tumor cell lines was epigenetically regulated by the methylation of $\mathrm{CpGs}$ in this promoter region. Many reports have described the epigenetic silencing of tumor-suppressor genes in cancer development, but few have described the epigenetic regulation of oncogenes (20). Loss of methylation might be common in cancer, but it is not well studied what genes are affected by this process. Here we found that ERas expression was affected by epigenetic modification of DNA promoter methylation. Our results provide an example for re-expression of an embryonic oncogene, ERas, in cancer cells by loss of methylation. These results suggest that DNA methylation might be the primary mechanism that actively modulates epigenetic transcriptional silencing of the ERas oncogene in human gastric carcinomas.

DNA methyltransferases were reported to regulate DNA methylation, however, no correlation was found between the ERas expression and the expression of DNA methyltransferases Dnmt1, Dnmt3a, and Dnmt3b, in this study. Dnmt1, Dnmt3a, and Dnmt3b might not be associated with epigenetic regulation of ERas oncogene. Other mechanisms might exist in the epigenetic regulation of the ERas oncogenes. ERas expression was silent again 4 days after DNA methyltransferase inhibitor treatment, which may suggest that these epigenetic changes are regulated by on-off reversible systems.

CSCs generate tumors that recapitulate the phenotypic heterogeneity found in the tumor. ERas oncogene supports the tumor-like propagation of ES cells (1). We hypothesized that cancer cells with ERas expression might have characteristics of CSC. In this study, we found that ERas expression correlates with SP frequency as a marker for tumor stem cells. Although all of the SP cells do not necessarily represent CSCs, SP cells might exhibit characteristics of a tumor-initiating CSC phenotype. The percentage of CSC like-SP fraction was $3.6 \%$ in ERas-positive gastric cancer cell lines, while that of
ERas-negative was $1.6 \%$. A previous study (6) reported that the percentage of SP fraction of the gastric cancer cell lines NUGC-3 and MKN-7, was $2.1 \%$ and $0.6 \%$, respectively. These percentages of SP fraction were similar to those of our study in NUGC-3 (2.41\%) and MKN-7 (1.66\%). These findings demonstrate that gastric cancer cells with ERas properties contain more SP cells that show some characteristics of so-called CSCs. ERas might be an important target for cancer therapy and a useful tool to identify the characteristics of CSCs.

In conclusion, the activating ERas might be associated with the tumorigenesis of gastric carcinomas, and might be one of molecules responsible for cancer stem cell-like characteristics. DNA promoter methylation of ERas might be the primary mechanism that actively modulates epigenetic transcriptional silencing of the ERas oncogene.

\section{Acknowledgements}

This study was supported in part by Grants-in-Aid for Scientific Research (nos. 18591475, 20591073, and 18390369) from the Ministry of Education, Science, Sports, Culture and Technology of Japan, by a Grant-in-Aid for the Japanese Society of Gastroenterology for Scientific Research, by a Grant-in-Aid for Kobayashi Foundation for Innovative Cancer Chemotherapy, by a Grant-in-Aid for the Sagawa Foundation for Cancer Research, and by a Grant-in-Aid for the Osaka Medical Research Foundation for Incurable Diseases. We thank Professor Shinya Yamanaka (Department of Stem Cell Biology, Institute for Frontier Medical Sciences, Kyoto University, Japan) for helpful advice.

\section{References}

1. Takahashi K, Mitsui K and Yamanaka S: Role of ERas in promoting tumour-like properties in mouse embryonic stem cells. Nature 423: 541-545, 2003.

2. Yashiro M, Nishioka N and Hirakawa K: K-ras mutation influences macroscopic features of gastric carcinoma. J Surg Res 124: 74-78, 2005.

3. Fang JY and Lu YY: Effects of histone acetylation and DNA methylation on $\mathrm{p} 21$ (WAF1) regulation. World J Gastroenterol 8: 400-405, 2002.

4. Plass C: Cancer epigenomics. Hum Mol Genet 11: 2479-2488, 2002.

5. Ballestar E and Esteller M: The epigenetic breakdown of cancer cells: from DNA methylation to histone modifications. Prog Mol Subcell Biol 38: 169-181, 2005.

6. Robertson KD: DNA methylation, methyltransferases, and cancer. Oncogene 20: 3139-3155, 2001.

7. Santini V, Kantarjian HM and Issa JP: Changes in DNA methylation in neoplasia: pathophysiology and therapeutic implications. Ann Intern Med 134: 573-586, 2001.

8. Haraguchi N, Utsunomiya T, Inoue H, Tanaka F, Mimori K, Barnard GF and Mori M: Characterization of a side population of cancer cells from human gastrointestinal system. Stem Cells 24: 506-513, 2006.

9. Ho MM, Ng AV, Lam S and Hung JY: Side population in human lung cancer cell lines and tumors is enriched with stemlike cancer cells. Cancer Res 67: 4827-4833, 2007.

10. Wang J, Guo LP, Chen LZ, Zeng YX and Lu SH: Identification of cancer stem cell-like side population cells in human nasopharyngeal carcinoma cell line. Cancer Res 67: 3716-3724, 2007.

11. Szotek PP, Pieretti-Vanmarcke R, Masiakos PT, Dinulescu DM, Connolly D, Foster R, Dombkowski D, Preffer F, MacLaughlin DT and Donahoe PK: Ovarian cancer side population defines cells with stem cell-like characteristics and mullerian inhibiting substance responsiveness. Proc Natl Acad Sci USA 103: 11154-11159, 2006. 
12. Goodell MA, Brose K, Paradis G, Conner AS and Mulligan RC: Isolation and functional properties of murine hematopoietic stem cells that are replicating in vivo. J Exp Med 183: 1797-1806, 1996.

13. Yasuda K, Yashiro M, Sawada T, Ohira M and Hirakawa K: ERas oncogene expression and epigenetic regulation by histone acetylation in human cancer cells. Anticancer Res 27: 4071-4075, 2007.

14. Yashiro $M$, Chung YS, Nishimura $S$, Inoue $T$ and Sowa $M$ : Establishment of two new scirrhous gastric cancer cell lines: analysis of factors associated with disseminated metastasis. Br J Cancer 72: 1200-1210, 1995.

15. Yashiro M, Chung YS, Nishimura S, Inoue $\mathrm{T}$ and Sowa $\mathrm{M}$ : Peritoneal metastatic model for human scirrhous gastric carcinoma in nude mice. Clin Exp Metastasis 14: 43-54, 1996.

16. Murahashi K, Yashiro M, Takenaka C, Matsuoka T, Ohira M and Chung KH, Establishment of a new scirrhous gastric cancer cell line with loss of heterozygosity at E-cadherin locus. Int J Oncol 19: 1029-1033, 2001.
17. Takemura S, Yashiro M, Sunami T, Tendo M and Hirakawa K: Novel models for human scirrhous gastric carcinoma in vivo. Cancer Sci 95: 893-900, 2004.

18. Sakai N, Maruyama T, Sakurai R, Masuda H, Yamamoto Y, Shimizu A, Kishi I, Asada H, Yamagoe S and Yoshimura Y: Involvement of histone acetylation in ovarian steroid-induced decidualization of human endometrial stromal cells. J Biol Chem 278: 16675-16682, 2003.

19. Yashiro M, Carethers JM, Laghi L, Saito K, Slezak P, Jaramillo E, Rubio C, Koizumi K, Hirakawa K and Boland CR: Genetic pathways in the evolution of morphologically distinct colorectal neoplasms. Cancer Res 61: 2676-2683, 2001.

20. Esteller M, Toyota M, Sanchez-Cespedes M, Capella G, Peinado MA, Watkins DN, Issa JP, Sidransky D, Baylin SB and Herman JG: Inactivation of the DNA repair gene $\mathrm{O}^{6}$-methylguanine-DNA methyltransferase by promoter hypermethylation is associated with $\mathrm{G} \rightarrow \mathrm{A}$ mutations in K-ras in colorectal tumorigenesis. Cancer Res 60: 2368-2371, 2000. 